\title{
Adaptive Nulling in Monopulse Antennas
}

\author{
RANDY L. HAUPT, MEMBER, IEEE
}

\begin{abstract}
A method for phase-only adaptive nulling in monopulse antennas is presented, using a gradient search algorithm. The array's beam steering phase shifters double as the adaptive weights. Both computed and experimental results are presented.
\end{abstract}

\section{INTRODUCTION}

A MONOPULSE radar antenna simultaneously forms sum and difference beams through two separate beam-forming networks. Combining these beams in special ways provides information for accurately detecting and tracking a target. The two corporate feeds in a monopulse phased array considerably complicate the design over a phased array with a single corporate feed. Low sidelobe and adaptive nulling requirements further increase the antenna complexity. Thus there is motivation for simplifying the adaptive nulling process in monopulse phased arrays.

Most adaptive nulling algorithms require variable complex weights at each element in the array. Although complex adaptive weights offer excellent control over the antenna pattern, most phased arrays are equipped with only phase controls. Adding variable amplitude weights considerably increases the cost but is becoming more common as the demands on antenna performance become greater. Placing the adaptive weights at the subarray outputs offers some economical advantages, but grating lobes limit this form of implementation [1].

Several studies have addressed phase only nulling in lowsidelobe antennas [2]-[4]. Phase-only nulling has the advantage of a simple design. Beam-steering phase shifters (which are already part of the antenna) provide the means of generating nulls in the antenna pattern. As long as the adaptive phase shifts are small, the low sidelobes are maintained and the main beam remains unchanged.

Usually, an adaptive nulling algorithm considers only the sum channel. These algorithms also apply to monopulse antennas if there are separate adaptive weights for the sum and difference channels. Rather than building separate adaptive processes for each channel, a simple design results when the two channels share the adaptive weights. Such a technique is theoretically feasible for amplitude and phase nulling as well as phase-only nulling [5]. More recently, $\mathrm{Vu}$ described a technique for simultaneous nulling in the sum and difference patterns with variable-amplitude weights [6].

This paper presents theoretical and experimental results of phase-only nulling in low-sidelobe monopulse antennas. Both

Manuscript received September 24, 1986; revised April 17, 1987.

The author was with RADC/EEC, Hanscom Air Force Base, MA. He is now with the Department of Electrical Engineering, United States Air Force Academy, Colorado Springs, CO 80840.

IEEE Log Number 8718300 . results are based on a gradient search algorithm that simultaneously searches for a minimum in the sum and difference channel output powers. Some of the experimental results were discussed previously [7].

\section{Phase-Only Gradient Search Algorithm for a Low-Sidelobe Monopulse ARRAY}

The goal of a phase-only adaptive algorithm is to find a phase shift $\Psi$ to minimize that part of the output power $(P)$ due to the undesired signals while not seriously changing the output power due to the desired signals. The equation for the output power of a linear array of $N$ isotropic sources with $M$ incident plane waves due to signal sources in the far field is given by

$$
\begin{aligned}
P & =P(A, X, \Psi, Q, S, \theta) \\
& =\left|\sum_{m=1}^{M} \sum_{n=1}^{N} \gamma_{m n} a_{n} e^{j\left(2 \pi x_{n} \sin \theta_{m}-\psi_{n}-q_{n}-s_{n}\right)}\right|^{2}
\end{aligned}
$$

where

$$
\begin{aligned}
A= & {\left[a_{1}, a_{2}, \cdots, a_{N}\right], \text { amplitude weight vector, } } \\
X= & {\left[x_{1}, x_{2}, \cdots, x_{N}\right], \text { element spacing in wavelengths, } } \\
\Psi= & {\left[\psi_{1}, \psi_{2}, \cdots, \psi_{N}\right], \text { adaptive weight settings, } } \\
Q= & {\left[q_{1}, q_{2}, \cdots, q_{N}\right], \text { quiescent phase shifter settings for } } \\
& \text { optimum gain, }
\end{aligned}
$$

$S=\left[s_{1}, s_{2}, \cdots, s_{N}\right]$, beam-steering phase settings,

$\gamma_{m n} \quad$ electric field at element $n$ due to signal $m$ in the far field,

$\theta_{m} \quad$ direction of signal $m$ from broadside,

$N$ number of elements in the array,

$M$ number of desirable and undesirable signals in the far field.

Note that the adaptive phase shifts just superimpose on the quiescent and beam-steering phase shifter settings. As a result, only one set of phase shifters performs all three functions.

Many possible search algorithms exist for finding $\Psi$. The algorithm used here is the gradient search or steepest descent algorithm. This algorithm is a well-known method of solving nonlinear problems. It forms the basis for many advanced algorithms and is often used as a standard by which to compare other algorithms.

The equation for the gradient search algorithm is

$$
\Psi^{(k+1)}=\Psi^{(k)}-\alpha \nabla P\left(\Psi^{(k)}\right)
$$

where the gradient of $P$ is given by

$\nabla P\left(\Psi^{(k)}\right)=\left[\frac{\partial P\left(\Psi^{(k)}\right)}{\partial \psi_{1}}, \frac{\partial P\left(\Psi^{(k)}\right)}{\partial \psi_{2}}, \cdots, \frac{\partial P\left(\Psi^{(k)}\right)}{\partial \psi_{N}}\right]$. 
In discrete form, the $n$th component of the gradient is

$$
\frac{\partial P}{\partial \psi_{n}}=\frac{\Delta P_{n}}{\Delta \psi}
$$

where $\Delta P_{n}$ is the change in output power due to $\Delta \psi$, and $\Delta \psi$ is the phase shifter step size, a constant.

The derivative in this equation may be represented by a central or forward difference formula. Surprisingly, the central difference formula did not improve the algorithm's performance. In fact, since two power measurements are necessary to form the central difference derivative (versus only one power measurement for the forward difference derivative), the central difference method is considerably slower. Therefore, the algorithm described here uses the forward differencing scheme.

The standard mathematical approaches to minimizing a function start by assuming the function is quadratic. When the function is not quadratic, little can be said about the convergence properties of the algorithm.

The output power as a function of phase shifter setting does not form a quadratic surface. In fact, many combinations of phase shifter settings will form a null (find a local minimum in the output power) at any given angle. The best combination forms the nulls in the directions of the undesired signals, with a minimum loss in gain and a minimum perturbation to the sidelobe level. This objective is feasible when few undesirable signals exist and the adaptive phase settings are kept to the minimum.

The adaptive weights may be thought of as random perturbations to the phase taper of the array (although not true for all adaptive nulling algorithms, this statement is fairly accurate for this algorithm). Consequently, the sidelobe level is proportional to the size of the phase perturbations and inversely related to the number of elements. By keeping the adaptive phase shifts small, the average sidelobe level and the main beam gain do not drastically change.

The phase shifter settings may be kept small by finding an $\alpha$ that satisfies (1). An $\alpha$ that satisfies

$$
P(x-\alpha \nabla P)=\text { minimum }
$$

may be found by doing a line search along the gradient $\nabla P$. However, a line search is slow and may require many power measurements. Also, no limit exists on the value of $\alpha$, hence no limit to the size of perturbations to the antenna pattern. The $\alpha$ used in this paper normalizes the gradient and limits the step size taken along the gradient to a value of $\Delta \psi$ :

$$
\alpha=\frac{\Delta \psi}{\sqrt{\left(\sum_{n=1}^{N}\left(\frac{\Delta P_{n}}{\Delta \psi}\right)^{2}\right)} .}
$$

If $\Delta \psi$ is carefully chosen, then the desired nulls will form in the pattern without significantly disturbing the rest of the pattern. A small value of $\Delta \psi$ may not produce the deepest nulls, but it will form a more accurate approximation to the gradient (4) and will not perturb the pattern much. On the other hand, too small a value of $\Delta \psi$ will not adequately form the nulls and will converge very slowly.

The algorithms begins by assigning a constant value to $\Delta \psi$. For instance, an eight-bit phase shifter has a possible 256 settings. The increment between settings is $1.4^{\circ}$. For one to three nulls and $N>20$, a good starting value for $\Delta \psi$ is $7^{\circ}$. Each element in the gradient is found by changing a phase shifter setting by $\Delta \psi$ and measuring the change in output power. Then the phase shifter is restored to its original value, and the process repeated for all the remaining array phase shifters. The algorithm iterates as long as $\Psi^{(k+1)}$ reduces the total output power. If the output power does not go down, then $\Delta \psi$ is decremented by one setting $\left(1.4^{\circ}\right)$ and the iteration is started again. The algorithm stops when $\Delta \psi=0^{\circ}$.

The value for $\Delta P_{n}$ in (4) may be found in several ways. If only one of the channel output powers is considered, then either one of the following is possible:

$$
\begin{gathered}
\text { sum channel: } \Delta P_{n}=P S-P S_{n} \\
\text { difference channel: } \Delta P_{n}=P D-P D_{n}
\end{gathered}
$$

where

PS output power of sum channel due to phase settings $\Psi^{(0)}$,

$P S_{n} \quad$ output power of sum channel when phase shifter $n$ is incremented by $\Delta \psi$,

$P D$ output power of difference channel due to phase settings $\Psi^{(0)}$,

$P D_{n}$ output power of difference channel when phase shifter $n$ is incremented by $\Delta \psi$.

Either of these equations will place a null in its respective channel's antenna pattern but will not simultaneously place the null in both patterns. A more proper choice considers the change in output power of both channels. Two possible selections are

$$
\begin{aligned}
& \Delta P_{n}=\sqrt{P S_{n} * P D_{n}-P S * P D} \\
& \Delta P_{n}=\left(P S_{n}+P D_{n}\right)-(P S+P D) .
\end{aligned}
$$

After trying these equations in the computer simulation and the experiment, I can find no preference for one over the other.

Checking to see whether the adaptive phase settings lowered the output power may be done in several ways. First, one may require that either one or both of the channel output powers go down:

$$
\begin{gathered}
S P^{(1)}<S P^{(0)} \\
D P^{(1)}<D P^{(0)} \\
S P^{(1)}<S P^{(0)} \text { and } D P^{(1)}<D P^{(0)} \\
S P^{(1)}<S P^{(0)} \text { or } D P^{(1)}<D P^{(0)}
\end{gathered}
$$

where

$S P^{(0)} \quad$ output power of the sum channel due to $X^{(0)}$,

$S P^{(1)}$ output power of the sum channel due to $X^{(1)}$,

$D P^{(0)}$ output power of the difference channel due to $X^{(0)}$,

$D P^{(1)}$ output power of the difference channel due to $X^{(1)}$. 


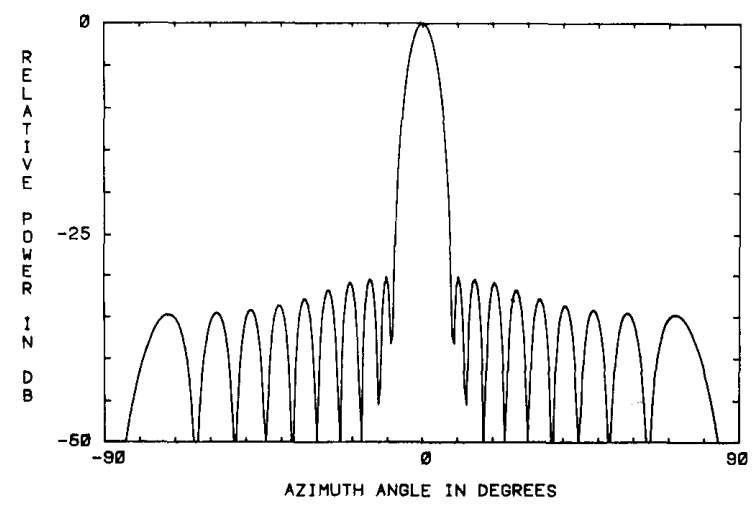

(a)

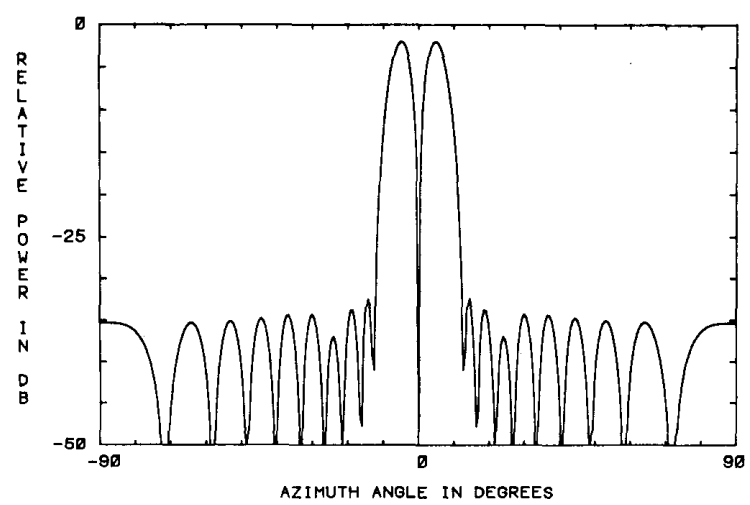

(b)

Fig. 1. Quiescent far-field patterns of linear array of 20 isotropic elements. (a) Sum channel. (b) Difference channel.

Equations (10)-(12) usually result in a null in only one of the patterns, but not both. At the opposite extreme (12), when the requirement is that both channel output powers must go down, then the nulls are poorly formed.

A much better check to determine whether the output power goes down involves some combination of the output powers of both channels. Two examples are

$$
\begin{aligned}
& S P^{(1)} * D P^{(1)}<S P^{(0)} * D P^{(0)} \\
& S P^{(1)}+D P^{(1)}<S P^{(0)}+D P^{(0)} .
\end{aligned}
$$

Either of these choices lead the gradient search algorithm to put nulls in both the sum and difference patterns.

\section{COMPUTER Results}

The computer model has 20 isotropic elements, each having its own eight-bit phase shifter. The sum channel has a lowsidelobe Taylor amplitude taper, and the difference channel has a low-sidelobe Bayliss taper. These tapers produce the quiescent sum and difference patterns shown in Figs. 1(a) and 1 (b).

The computer model has several options for $\Delta P_{n}$ and the performance criteria. Equations (6), (7), (8), or (9) are the choices for $\Delta P_{n}$, and any equation from (10) through (15) is the choice for the adaptive performance criterion. The starting value for $\Delta \psi$ is $7^{\circ}$. Table I lists the results from adaptive nulling in the presence of three interference sources at $-35^{\circ}$, $25^{\circ}$, and $61^{\circ}$. The quiescent condition is normalized to $0-\mathrm{dB}$ output. The first column is the equation number that defines $\Delta P_{n}$. The second column is the equation number of the adaptive performance criterion. Finally, the last two columns show the change in output power in the sum and difference channels due to the adaptive phase settings. Figs. 2(a) and 2(b) show the resulting adapted far-field pattern with (5) as the gradient and (14) to judge the performance.

Another technique produced similar final results as shown in Table I. This method consists of alternating the adaptive nulling algorithm between the sum and difference channels. In other words, nulls are adaptively placed in the sum channel using (3) for the gradient and (14) as the convergence criterion. Next, the phase shifters are not reset, but the adaptive nulling algorithm places nulls in the difference channel using (4) for the gradient and (10) as the convergence criterion. Returning to the sum channel, the process repeats until the desired nulls appear in both patterns. Figs. 3(a) and 3 (b) are the resulting far-field patterns for interference sources at $-35^{\circ}, 25^{\circ}$, and $61^{\circ}$. The second method works fine but takes longer than the first method. 


\begin{tabular}{|c|c|c|c|}
\hline \multirow{3}{*}{$\begin{array}{l}\text { Gradient } \\
\text { Equation }\end{array}$} & \multicolumn{3}{|c|}{$\begin{array}{c}\text { TABLE I } \\
\text { SIMULATED PERFORMANCE OF ADAPTED ARRAY }\end{array}$} \\
\hline & \multirow{2}{*}{$\begin{array}{l}\text { Convergence } \\
\text { Criterion }\end{array}$} & \multirow{2}{*}{$\begin{array}{c}\text { Sum Channel } \\
\text { Relative Power (dB) }\end{array}$} & \multirow{2}{*}{$\begin{array}{l}\text { Difference Channel } \\
\text { Relative Power (dB) }\end{array}$} \\
\hline & & & \\
\hline \multicolumn{2}{|c|}{ Quiescent Patterns } & 0 & 0 \\
\hline 3 & 9 & -24.4 & -4.6 \\
\hline 3 & 10 & -15.8 & -9.9 \\
\hline 3 & 11 & -24.2 & -5.4 \\
\hline 3 & 12 & -20.1 & -6.8 \\
\hline 3 & 13 & -24.4 & -4.6 \\
\hline 3 & 14 & -17.3 & -9.8 \\
\hline 4 & 9 & -2.0 & -15.2 \\
\hline 4 & 10 & -2.3 & -19.4 \\
\hline 4 & 11 & -2.3 & -19.4 \\
\hline 4 & 12 & -3.6 & -20.9 \\
\hline 4 & 13 & -2.3 & -19.4 \\
\hline 4 & 14 & -2.0 & -15.2 \\
\hline 5 & 9 & -20.6 & -11.5 \\
\hline 5 & 10 & -11.9 & -15.1 \\
\hline 5 & 11 & -11.9 & -15.1 \\
\hline 5 & 12 & -20.9 & -14.0 \\
\hline 5 & 13 & -21.9 & -14.3 \\
\hline 5 & 14 & -17.6 & -15.1 \\
\hline 6 & 9 & -21.5 & -12.7 \\
\hline 6 & 10 & -16.7 & -20.6 \\
\hline 6 & 11 & -16.7 & -20.6 \\
\hline 6 & 12 & -16.71 & -17.6 \\
\hline 6 & 13 & -16.7 & -20.6 \\
\hline 6 & 14 & -22.3 & -18.9 \\
\hline
\end{tabular}

\section{EXPERIMENTAL RESULTS}

Details about the antenna and test range appear in [11]. Consequently, only a brief sketch of the experiment is related here. The antenna is a linear array of 80 elements with eightbit phase shifters. Its sum and difference channels have lowsidelobe Taylor and Bayliss amplitude tapers, respectively. Fig. 4 shows a picture of the antenna. Its quiescent sum and difference patterns appear in Figs. 5(a) and 5(b).

The measurements were performed at the RADC far-field test range in Ipswich, MA. Nulling was done in the sidelobes of the antenna using a single $\mathrm{CW}$ source as the interference. Although not a realistic situation, this experiment is one step beyond a computer simulation. The antenna was positioned such that a sidelobe in both the sum and difference patterns pointed toward the source. Next, the algorithm was turned on. When the algorithm stopped, the adaptive phase shifters were frozen. Then the far-field patterns were recorded.

Figs. 6(a) and 6(b) and 7(a) and 7(b) are the far-field adapted patterns when $\Delta \psi$ is started at $7^{\circ}$. Figs. 6(a) and 6(b) simultaneously considered both the sum and difference patterns by using (5) and (14). This figure corresponds to Figs. 2(a) and 2(b) of the computer simulation. The source is at $23^{\circ}$. Figs. 7(a) and 7(b) show the results of nulling in alternating channels as was done in the computer simulation (Figs. 3(a), 3(b)). In this case the source is at $5.1^{\circ}$. Notice the deep nulls formed in the sidelobes. In addition, the rest of the far-field pattern was virtually undisturbed. The main beam gain of the adapted patterns did not change from the quiescent gain when one undesirable signal existed in the far field. The main beam peaks in Figs. 6(a) to 7(b) are normalized to the main beam peaks of their respective quiescent patterns in Figs. 5(a) and 5 (b).

\section{CONCLUSION}

This paper presents a gradient search algorithm for simultaneously placing nulls in the low-sidelobe sum and difference patterns of a monopulse antenna. Such an algorithm has the potential for being used on phased arrays already in existence since it uses the beam-steering phase shifters as the adaptive weights.

Low-sidelobe antennas are designed at great expense to reduce interference signals at all angles outside the main beam. Hence the adaptive nulling process must strive to maintain the low-sidelobe levels while placing nulls in the directions of interference sources. The more jamming signals present, the more difficult it is to maintain the sidelobe level. A proper choice of the phase increment $\Delta \psi$ is very important. When the number of jammers is small compared to the number of elements and $\Delta \psi$ is of modest size, the gain and average sidelobe levels remain virtually unchanged.

The important steps in the algorithm are deciding how to calculate the change in output power and how to judge the performance of the algorithm after each iteration. In both cases the sum and difference channel output powers must be combined in some fashion to produce successful results.

As demonstrated by the computer program and the experiment, this algorithm is capable of placing very deep nulls in the antenna patterns in the directions of interference. Although the algorithm successfully places nulls in both patterns, it is slow. No attempt was made to streamline the algorithm or find faster methods of convergence. The goal of this project was 


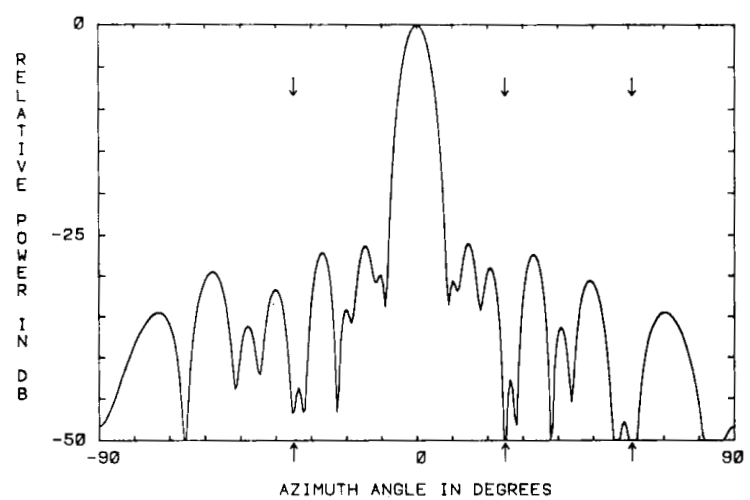

(a)

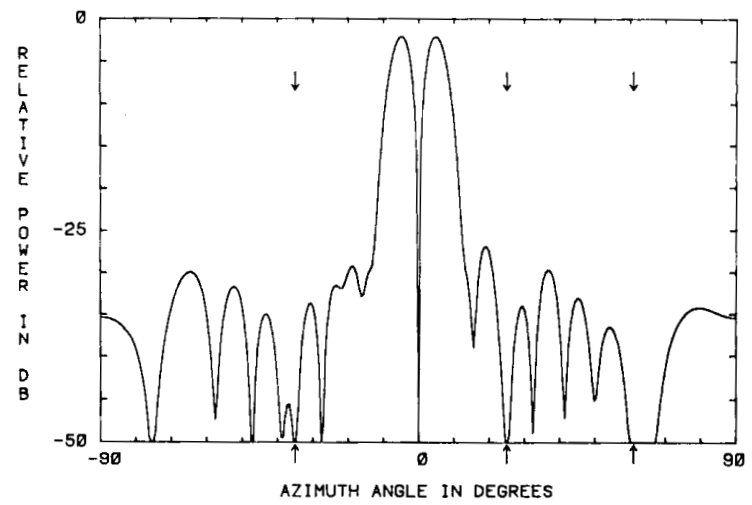

(b)

Fig. 2. Adaptive nulls were placed in these far-field patterns by simultaneously nulling in sum and difference channels using (5) and (14). Interference sources are at $-35^{\circ}, 25^{\circ}$, and $61^{\circ}$. (a) Sum channel. (b) Difference channel.

not to develop new algorithms but to demonstrate the feasibility of simultaneously placing adaptive nulls in the sum and difference patterns of an experimental low-sidelobe monopulse array.

Some last words about the inherent main beam constraint are needed. First, the small values of $\Delta \psi$ will not destroy the main beam gain. I tried using this algorithm to place a null in the main beam. The algorithm was only able to alter the main beam when the values of $\Delta \psi$ were very large. I have found that a reasonably small value of $\Delta \psi$ will inherently constrain the main beam and sidelobe levels. Second, the jamming signal is often always present, whereas the returning radar signal is only present for a short time. This implies that the adaptive nulling usually takes place when only the jamming signal is present. As a result, the algorithm spends little time trying to null the desired signal.

I was hoping to present much more extensive experimental results with multiple wide-band jammers, but an accident prevented any further work. A high-performance antenna is a very delicate piece of equipment and must be handled like a baby. Unfortunately, the baby was dropped several feet onto the antenna positioner when being put into place with a crane. Fortunately, I did have several experimental results, some of

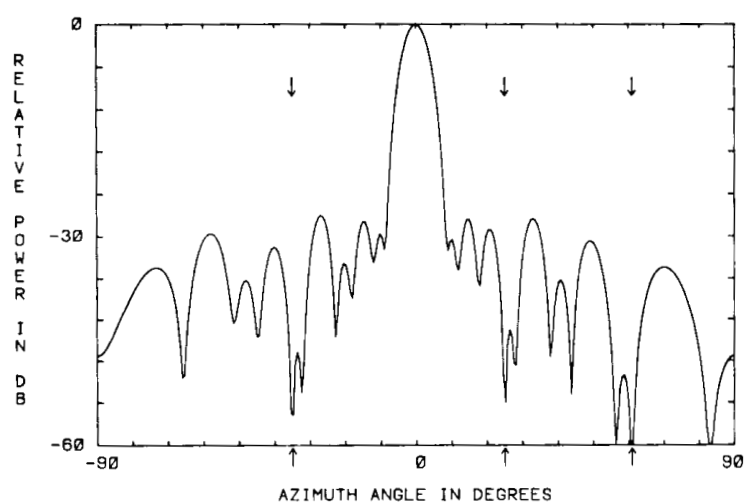

(a)

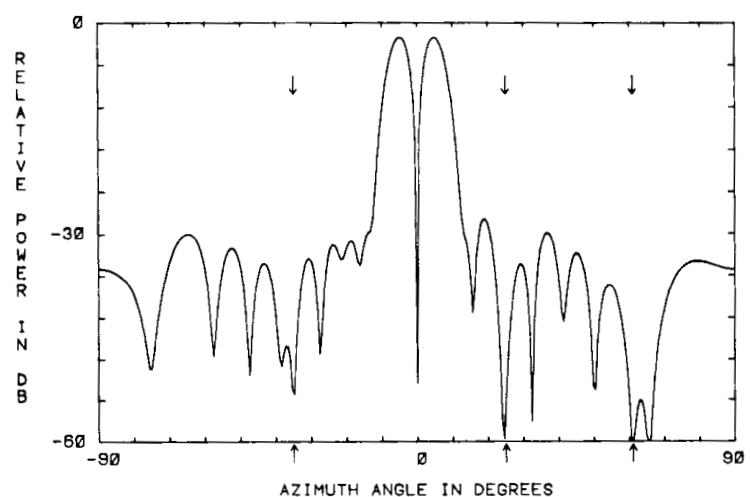

(b)

Fig. 3. Adaptive nulls were placed in these far-field patterns by nulling first in sum channel, then in difference channel and sequence repeated three times. Interference sources are at $-35^{\circ}, 25^{\circ}$, and $61^{\circ}$. (a) Sum channel. (b) Difference channel.

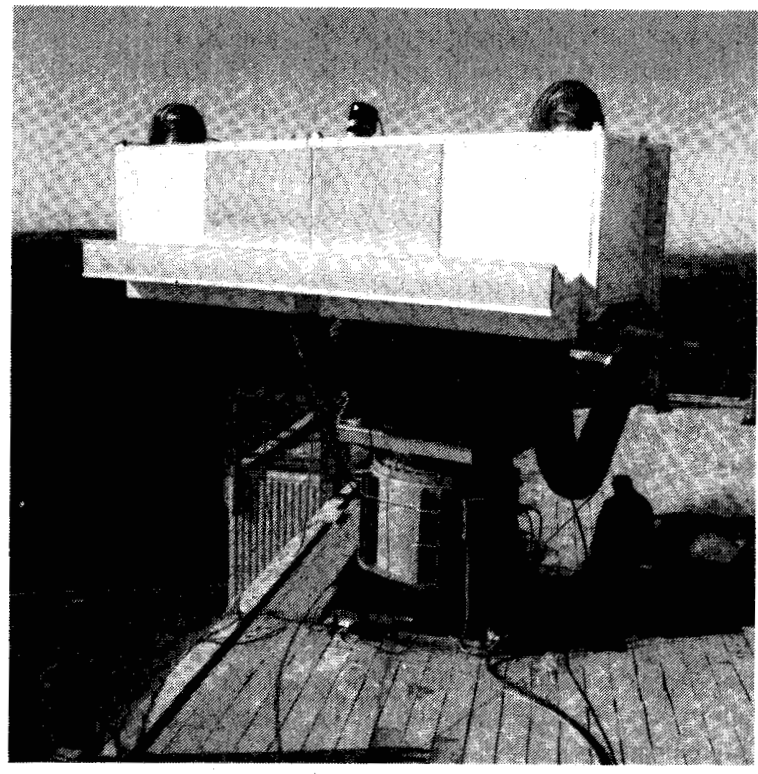

Fig. 4. Picture of experimental adaptive nulling linear array. 
HAUPT: ADAPTIVE RULING IN MONOPULSE ANTENNAS

207
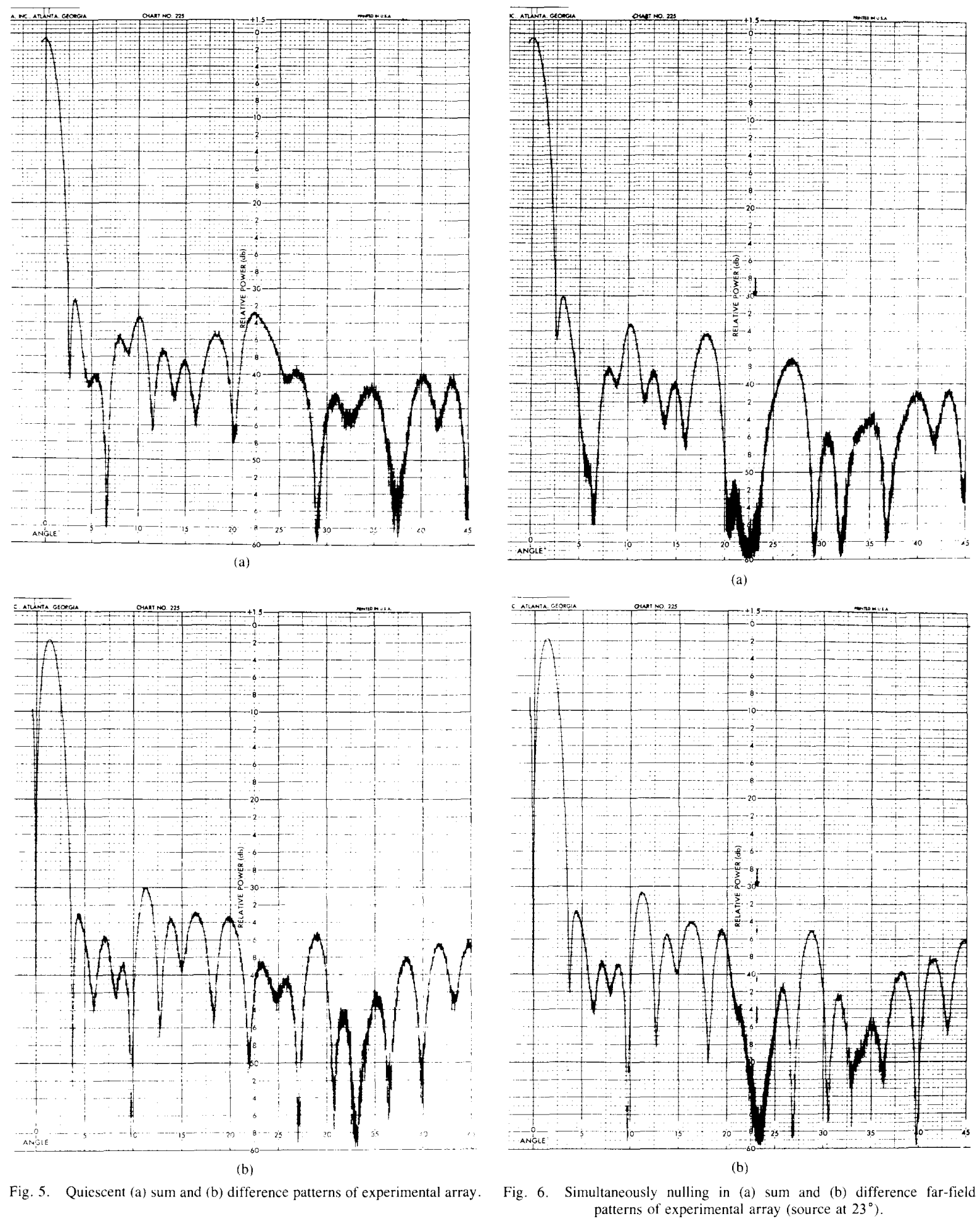
patterns of experimental array (source at $23^{\circ}$ ). 


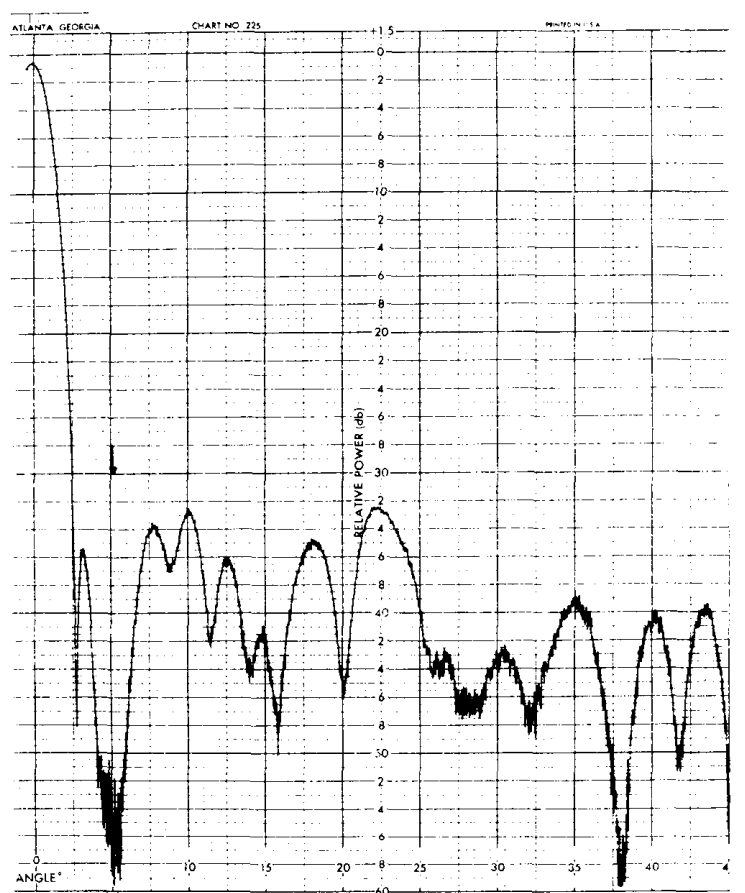

(a)

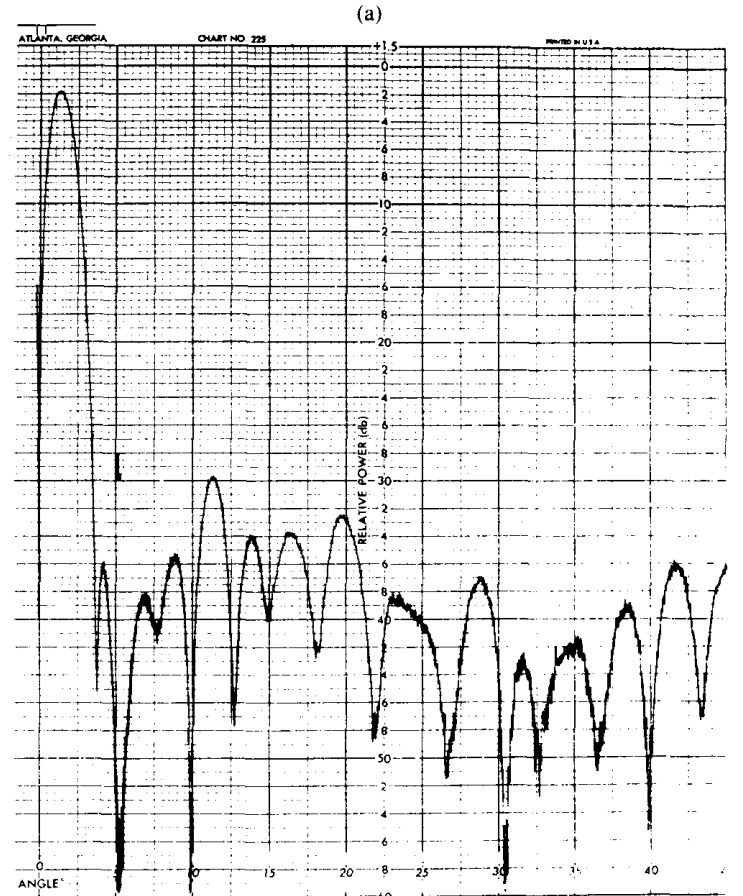

(b)

Fig. 7. Nulling in far-field patterns one at a time with source at $5 \cdot 1^{\circ}$. (a) Sum channel. (b) Difference channel. which are presented here, before this tragic accident happened.

\section{ACKNOWLEDGMENT}

I want to thank Livio Poles and Garret Murdza for their many hours of work spent setting up the adaptive nulling experiments. I also owe many thanks to Lt. Col. Michael O'Brien, Dr. Robert Shore, and Dr. J. Leon Poirer for their technical help and encouragement in this project. Finally, I want to thank Dr. Martin Herman for reviewing the revised paper.

\section{REFERENCES}

[1] R. L. Haupt, "Null synthesis with phase and amplitude controls at the subarray outputs," IEEE Trans. Antennas Propagat., vol. AP-33, pp. 505-509, May 1985.

[2] H. Steyskal, "Simple method for pattern nulling by phase perturbation," IEEE Trans. Antennas Propagat., vol. AP-31, pp. 163-166, Jan. 1983.

[3] R. Shore and H. Steyskal, "Nulling in linear array patterns with minimization of weight perturbations," RADC-TR-82-32 (ADA118695), Feb. 1982.

[4] C. Baird and G. Rasswiler, "Adaptive sidelobe nulling using digitally controlled phase shifters," IEEE Trans. Antennas Propagat., vol. AP-24, pp. 638-649, Sept. 1976.

[5] R. L. Haupt, "Simultaneous nulling in the sum and difference patterns of a monopulse array," IEEE Trans. Antennas Propagat., vol. AP32, pp. 486-493, May 1984.

[6] T. B. Vu, "Simultaneous nulling in sum and difference patterns by amplitude control," IEEE Trans. Antennas Propagat., vol. AP-34, pp. 214-218, Feb. 1986.

[7] R. L. Haupt, "Adaptive nulling in monopulse antennas," in IEEE Antennas Propagat. Soc. Int. Symp. Dig., June 1984, pp. 819-822.

[8] H. Steyskal, R. A. Shore, and R. L. Haupt, "Methods for null contro] and their effects on the radiation pattern," IEEE Trans. Antennas Propagat., vol. AP-34, pp. 404-409, Mar. 1986.

[9] L. J. Griffiths, "Adaptive monopulse beamforming," Proc. IEEE, vol. 64, pp. 1260-1261, Aug. 1976.

[10] R. L. Burden and J. D. Faires, Numerical Analysis. Boston, MA: PWS Publishers, 1985

[11] R. L. Haupt and L. D. Poles, "Performance measurements of a low sidelobe adaptive nulling array," in Proc. Antenna Measurement Techniques Assoc. Meet., Sept. 1983, pp. 2.1-2.17.

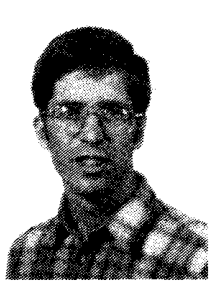

Randy L. Haupt (S'82-S'84-M'87) was born in Johnstown, PA, on August 11, 1956. He received the B.S. degree in electrical engineering from the United States Air Force Academy, Colorado Springs, $\mathrm{CO}$, the M.S. degree in engineering management from Western New England College, Springfield, MA, the M.S. degree in electrical engineering from Northeastern University, Boston, $\mathrm{MA}$, and the Ph.D. degree in electric engineering from The University of Michigan, Ann Arbor, in $1978,1981,1983$, and 1987, respectively.

From 1978 to 1980 he worked as an engineer in the Over-the-Horizon Radar System Program Office at Hanscom Air Force Base, MA. From 1980 to 1984 he worked on low sidelobe phased array and adaptive array research at the Electromagnetic Sciences Division of Rome Air Development Center at Hanscom AFB.

Dr. Haupt is a registered Professional Engineer in Colorado and a member of Tau Beta Pi. 